UDC 52, 532.1

\author{
K. Boshkayev \\ IETP, Physical-Technical Department of Al-Farabi Kazakh National University, \\ Al Farabi av. 71, 050040 Almaty, Kazakhstan \\ Dipartimento di Fisica and ICRA, Università di Roma "La Sapienza", \\ Piazzale Aldo Moro 5, I-00185 Roma, Italy \\ e-mail: kuantay@mail.ru
}

\title{
Non-rotating and slowly rotating stars in classical physics
}

\begin{abstract}
Equations are given for the calculation of the equilibrium configurations of slowly rotating stars in the framework of classical physics. In particular, prescriptions have been given to find the mass-radius, the mass-central density relations and the shapes of rotating stars. The equations which determine the relations between mass, central density and radius of rotating configurations take the form of an equation of hydrostatic equilibrium. These equations show the balance between the pressure, gravitational, and centrifugal forces correctly to second order in the angular velocity, but no other approximation is made. The equations which determine the moment of inertia and the quadrupole moment of the rotating star have also been derived.
\end{abstract}

Keywords: Hartle's formalism, equilibrium configurations, moment of inertia and quadrupole moment.

\section{Introduction}

In physics rotation may introduce a lot of changes in any system. For celestial object such as stars and planets their rotation plays a crucial role. Rotation does not only change the shape of the celestial objects but also influences the processes occurring inside stars i.e. it may accelerate or decelerate thermonuclear reactions in certain conditions, it changes the gravitational field outside the objects and it is one of the main factors that defines the lifespan of all stars (giant stars, main sequence, white dwarfs, neutron stars etc.) [1-3].

For instance, let us consider a white dwarf. A non-rotating white dwarf has a limiting mass $1.44 M_{\odot}$ which iswell-known as the Chandrasekhar limit [4]. The central density and pressure corresponding to this limit define future evolution of white dwarfs. If the white dwarf rotates, then due to the centrifugal forces the central density and pressure decrease [5]. In order to recover the initial values of the central density and pressure of a rotating star one needs to add extra mass. Here we see that a rotating star with the same central density and pressure as those of the non-rotating one possesses larger mass [6].

In this work we derive the equations describing the equilibrium configurations of slowly rotating stars within Hartle's formalism [7]. It is convenient to consider stars and planets as liquid when solving the problems of celestial mechanics, astronomy and astrophysics. The derived equations are valid with the precision to second order terms in the angular velocity for any liquid.

As a result we obtain the equations defining the main parameters of the rotating equilibrium configurations such as the mass, radius, moment of inertia, gravitational potential, angular momentum and quadrupole moment as a function of the central density and angular velocity (rotation period). In turn these parameters are of great importance in defining the further development - evolution of a star.

To pursue all these issues in more detail it is therefore of interest to determine what are the equilibrium configurations of a rotating star in classical physics. This is a problem, while presenting no difficulties of principle, is numerically complicated. Instead of one radial dimension, one has two or three dimensions. Instead of two ordinary differential equations to solve, one has the equivalent of an infinite system of ordinary differential equationsone for each coefficient of an expansion of all relevant quantities in spherical harmonics.

If, however, the star is rotating slowly, the calculation of its equilibrium properties is much simp-

Printed in Kazakhstan 
ler, because then the rotation can be considered as a small perturbation on an already-known nonrotating configuration. We therefore consider in this paper according to the Hartle formalism [7] the following problem:

- A one-parameter equation of state is specified: (pressure) $=($ known function of the density) $p=p(\rho)$, where $p$ is the pressure and $\rho$ is the density of matter. In general situations the pressure is also a function of temperature. This restricted form of the equation of state is appropriate when the temperature is a known function of the density inside the star. For example, this is the case when (1) all the matter is cold at the end point of thermonuclear evolution (see Harrison et al. 1965 [11]) or (2) when the star is in convective equilibrium so that changes in state are adiabatic (see, Chandrasekhar 1939 [10]).

- A non-rotating equilibrium configuration is calculated using this equation of state and the classical equation of hydrostatic equilibrium for spherical symmetry. The distribution of pressure, energy density, and gravitational field are thereby known.

- Axial and reflection symmetry. Attention is limited here to configurations which are axially symmetric. The configuration is symmetric about a plane perpendicular to the axis of rotation. From one's experience with the Newtonian theory of figures of equilibrium it is plausible that both of these assumptions are really consequences of the slow rotation of the configuration and not restrictions at all.

- This configuration is given a uniform angular velocity sufficiently slow so that the changes in pressure, energy density, and gravitational field are small. The configurations which minimize the total mass-energy (e.g., all stable configurations) must rotate uniformly (see Hartle and Sharp 1967 [12]).

- Slow rotation. From simple dimensional consideration this requirement implies

$$
\Omega^{2} \ll\left(\frac{c}{R}\right)^{2} \frac{G M}{R c^{2}}
$$

where $\Omega$ is the angular velocity of the star, $M$ is the mass of the unperturbed configuration, $R$ is its radius, $G$ is the gravitational constant, $c$ is the speed of light. For the unperturbed configuration the factor $G M / R c^{2}$ is less than unity $[8,9]$. Consequently the condition in equation (1) also implies

$$
R \Omega \ll c,
$$

- In other words, every particle must move at non-relativistic velocities if the perturbation of the geometry is to be small in terms of percentage.

- These small changes are considered as perturbations on the known non-rotating solution. The field equations are expanded in powers of the angular velocity and the perturbations calculated by retaining only the first-and second-order terms.

In this paper the equations necessary to solve this problem are obtained. Their numerical solution for particular equation of state and the analysis of the stability of the resulting configurations will be discussed in the forthcoming papers.

\section{Slowly rotating stars in Newtonian gravita- tional theory}

The theory of the equilibrium configurations of slowly rotating self-gravitating bodies has long been known in Newtonian gravitational theory (see, e.g., Jeffreys 1959 [13]; Chandrasekhar and Roberts 1963 [5]). In Newtonian gravitational theory the equilibrium values of pressure $p$, density $\rho$ and gravitational potential $\Phi$ of a fluid mass rotating with a uniform angular velocity $\Omega$ are determined by the solution of the three equations of Newtonian hydrostatic equilibrium. These are (1) the Newtonian field equation:

$$
\nabla^{2} \Phi(r, \theta)=4 \pi G \rho(r, \theta)
$$

(2) the equation of state which we have assumed to have a one-parameter form

$$
p=p(\rho)
$$

(3) the equation of hydrostatic equilibrium which can be summarized in the case of uniform rotation and a one-parameter equation of state by its first integral

$$
\begin{gathered}
\int_{0}^{p} \frac{d p(r, \theta)}{\rho(r, \theta)}-\frac{1}{2} \Omega^{2} r^{2} \sin ^{2} \theta \\
+\Phi(r, \theta)=\text { const. }
\end{gathered}
$$

The problem posed in the introduction to find the properties of a configuration of given central density and angular velocity can be phrased as follows. A solution $\Phi^{(0)}, p^{(0)}$, and $\rho^{(0)}$ of the Newtonian equations in the absence of rotation is known. This solution is the leading term in an ex- 
pansion of the solution including rotation in powers of the angular velocity $\Omega$. It is clear from the symmetry of the configuration under reversal of the direction of rotation that only even powers of the angular velocity will appear in this expansion. The equation of Newtonian hydrostatics are now expanded in powers of $\Omega^{2}$, and the equations which govern the second-order terms in the solution determined.

\section{Coordinate transformations}

Care must be exercised in choosing the coordinate system in which these expansions are carried out. For example, an expansion of the density as a function of the ordinary polar coordinates $r, \theta$ is not valid throughout the star. Such an expansion could be valid only if the fractional changes in density at each point in space were small. This condition cannot be met near the surface of the star as the surface of the configuration will be displaced from its non-rotating position and the perturbation in the density may be finite where the unperturbed density vanishes. To avoid this difficulty the points of space in the rotating configuration will not be labeled by the usual coordinates $r$ and $\theta$. Instead two coordinates $R$ and $\Theta$ defined as follows will be used: Consider a point inside the rotating configuration. This point

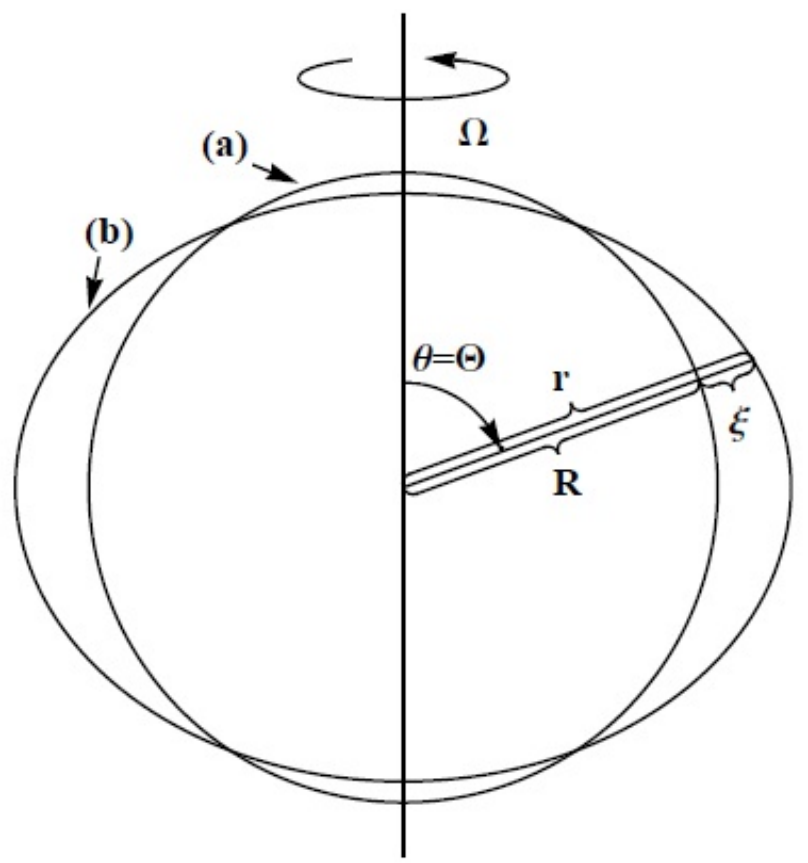

Figure 1 - Definition of the coordinates $R, \Theta$, and the displacement $\xi$.

The surface (a) is the surface of constant density $\rho(R)$ in the non-rotating configuration.

The surface (b) is the surface of constant density $\rho(R)$ in the rotating configuration.

lies on a certain surface of constant density. Ask for the radius of the surface in the non-rotating configuration which has precisely the same constant density. This radius is defined to be the coordinate $R$. The coordinate $\Theta$ is defined to be identical with the usual polar angle $\theta$. These definitions are given pictorially in Figure 1 and mathematically by the following equations:

$$
\Theta=\theta, \rho[r(R, \Theta), \Theta]=\rho(R)=\rho^{(0)}(R) .
$$

The function $r(R, \Theta)$ then replaces the density as a function to be calculated in the rotating configuration. The expansion of the $r(R, \Theta)$ in powers of the angular velocity will be written

$$
r \approx R+\xi+O\left(\Omega^{4}\right)
$$

The quantity $\xi=\xi(R, \Theta) \sim \Omega^{2}$ is the difference in radial coordinate $r$, between a point located by polar angle $\Theta$ on the surface of constant density $\rho(R)$ in the rotating configuration and the point located by the same polar angle on the surface of 
the same constant density on the non-rotating configuration (see Figure 1). For small angular velocities, the fractional displacement of the surface of constant density due to the rotation is small at the surface and in the middle of the star,

$$
\xi(R, \Theta) / R \ll 1
$$

It will also be small at the center of the star if the rotating configuration is chosen to have the same central density as the non-rotating configuration so that $\xi$ vanishes at $R=0$. We are always free to consider the rotating configuration as a perturbation on a non-rotating configuration of the same central density; so that equation (8) can be satisfied throughout the star. In the $R, \Theta$ coordinate system the two functions which characterize the rotating star are $r(R, \Theta)$ and the gravitational potential $\Phi(R, \Theta)$. The density and pressure are known functions of $R$ related by the equation of state

$$
\begin{gathered}
\rho[r(R, \Theta), \Theta]=\rho(R)=\rho^{(0)}(R), \\
p[r(R, \Theta), \Theta]=p(R)=p^{(0)}(R) .
\end{gathered}
$$

\section{Expansion in spherical harmonics}

The expansion of $r$ to terms in $\Omega^{2}$ is given by equation (7) and the expansion of $\Phi$ is denoted by

$$
\begin{gathered}
\Phi(R, \Theta) \approx \Phi^{(0)}(R)+\Phi^{(2)}(R, \Theta)+O\left(\Omega^{4}\right), \\
\Phi(r, \theta)=\Phi(R+\xi, \Theta) \approx \Phi(R, \Theta)+\xi \frac{d \Phi(R, \Theta)}{d R}+O\left(\Omega^{4}\right) \\
\approx \Phi^{(0)}(R)+\xi \frac{d \Phi^{(0)}(R)}{d R}+\Phi^{(2)}(R, \Theta)+O\left(\Omega^{4}\right) .
\end{gathered}
$$

These expansions are to be inserted in equations (3) and (5) written in the coordinates $R, \Theta$ with only terms of order $\Omega^{2}$ retained. The calculation of $\xi$ and $\Phi^{(2)}$ from the resulting equations is greatly simplified if these functions are first expanded in spherical harmonics since only a few terms in this series will remain in the final result. The reflection symmetry of the configuration implies that only spherical harmonics of even order will appear in this expansion if the polar axis is taken to be the axis of rotation.

$$
\begin{gathered}
\xi(R, \Theta)=\sum_{l=0}^{\infty} \xi_{l}(R) P_{l}(\cos \Theta), \\
\Phi^{(2)}(R, \Theta)=\sum_{l=0}^{\infty} \Phi_{l}^{(2)}(R) P_{l}(\cos \Theta), \\
\xi(R, \Theta), \Phi^{(2)}(R, \Theta) \sim \Omega^{2},
\end{gathered}
$$

where $P_{l}(\cos \Theta)$ are the Legendre polynomials. These expansions are to be substituted into the three equations of Newtonian hydrostatic equilibrium and the equations governing $\xi_{l}(R)$ and $\Phi_{l}^{(2)}$ derived.

When the expansions contained in equations (7), (10), and (11) are substituted into the integral of the equation of hydrostatic equilibrium (5), only those equations corresponding to the $l$ values 0 and 2 are found to contain the angular velocity, $\Omega$, in any way. This is because the centrifugal potential term in equation (5) has the angular dependence on $\sin ^{2} \Theta$. The Newtonian field equation when expanded in this way couples together only quantities with the same value of $l$. The equations for $\xi_{l}(R)$, $\Phi_{l}^{(2)}(R)$, with $l \geq 4$ are thus independent of $\Omega$ and their solution is

$$
\xi_{l}=0, \Phi_{l}^{(2)}=0, l \geq 4
$$

There remain only the quantities with $l=0$ and $l=2$ to be determined. This reduction in the number of $l$ values from infinity to 2 is the central simplification of the slow rotation approximation. In place of a system of partial differential equations one now only has ordinary differential equations for the four unknown functions $\Phi_{0}^{(2)}(R)$, $\Phi_{2}^{(2)}(R), \xi_{0}(R)$ and $\xi_{2}(R)$.

Now let us perform above-mentioned computations in detail. First we write down the Newtonian equation of hydrostatic equilibrium:

$$
\int_{0}^{p} \frac{d p(r, \theta)}{\rho(r, \theta)}-\frac{1}{2} \Omega^{2} r^{2} \sin ^{2} \theta+\Phi(r, \theta)=\text { const },
$$


then the definition of the Legendre polynomials:

$P_{0}(\cos \Theta)=1, P_{2}(\cos \Theta)=\frac{1}{2}\left(3 \cos ^{2} \Theta-1\right)$.

Now we will express $\sin ^{2} \Theta$ in terms of $P_{0}(\cos \Theta)$ and $P_{2}(\cos \Theta)$

$$
\sin ^{2} \Theta=\frac{2}{3}\left[P_{0}(\cos \Theta)-P_{2}(\cos \Theta)\right]
$$

From here we see that $l$ accepts only two values 0 and 2. Rewriting the Newtonian equation of hydrostatic equilibrium in coordinates $R, \Theta$ and expanding it in spherical harmonics we obtain

$$
\begin{gathered}
\int_{0}^{p} \frac{d p^{(0)}(R)}{\rho(R)}-\frac{1}{3} \Omega^{2} R^{2}\left[P_{0}(\cos \Theta)-P_{2}(\cos \Theta)\right]+\Phi^{(0)}(R) \\
+\sum_{l=0}^{n} \Phi_{l}^{(2)}(R) P_{l}(\cos \Theta)+\sum_{l=0}^{n} \xi_{l}(R) P_{l}(\cos \Theta) \frac{d \Phi^{(0)}(R)}{d R}=\text { const }
\end{gathered}
$$

Here we collect the equations $\sim \Omega^{0}$, and $\Omega^{2}$ with $l=0,2$ terms. The terms $\sim \Omega^{0}$ are given by

$$
\int_{0}^{p} \frac{d p^{(0)}(R)}{\rho(R)}+\Phi^{(0)}(R)=\text { constant }
$$

which corresponds to the Newtonian hydrostatic equation of a static configuration. Eventually terms $\sim \Omega^{2}$ with $l=0,2$ will be given by

$$
\begin{gathered}
-\frac{1}{3} \Omega^{2} R^{2}+\Phi_{0}^{(2)}(R)+\xi_{0}(R) \frac{d \Phi^{(0)}(R)}{d R}=0, \\
\frac{1}{3} \Omega^{2} R^{2}+\Phi_{2}^{(2)}(R)+\xi_{2}(R) \frac{d \Phi^{(0)}(R)}{d R}=0 .
\end{gathered}
$$

Using the same procedure the Newtonian field equation becomes

$$
\begin{array}{r}
\nabla^{2} \Phi(r, \theta)=\frac{1}{r^{2}} \frac{\partial}{\partial r}\left(r^{2} \frac{\partial \Phi(r, \theta)}{\partial r}\right)+\frac{1}{r^{2} \sin \theta} \frac{\partial}{\partial \theta}\left(\sin \theta \frac{\partial \Phi(\mathrm{r}, \theta)}{\partial \theta}\right) \\
=\nabla_{r}^{2} \Phi(r, \theta)+\frac{1}{r^{2}} \nabla_{\theta}^{2} \Phi(r, \theta) \approx \nabla_{r}^{2} \Phi^{(0)}(r)+\nabla_{r}^{2} \Phi_{0}^{(2)}(r) \\
+\nabla_{r}^{2} \Phi_{2}^{(2)}(r) P_{2}(\cos \theta)+\frac{1}{r^{2}} \nabla_{\theta}^{2} \Phi_{2}^{(2)}(r) P_{2}(\cos \theta)=4 \pi G \rho(r, \theta) .
\end{array}
$$

Knowing that functions $\Phi_{0}^{(2)}$ and $\Phi_{2}^{(2)}$ are already proportional to $\Omega^{2}$ we can directly write them in $(R, \Theta)$ coordinates. However the term $\nabla_{r}^{2} \Phi^{(0)}(r) \approx \nabla_{R}^{2} \Phi^{(0)}(R)+\xi(R, \Theta) \frac{d}{d R} \nabla_{R}^{2} \Phi^{(0)}(R)$ is defined this way, details are given in Appendix. Thus

$$
\begin{gathered}
\nabla^{2} \Phi(r, \theta)=\nabla_{R}^{2} \Phi^{(0)}(R)+\xi(R, \Theta) \frac{d}{d R} \nabla_{R}^{2} \Phi^{(0)}(R) \\
+\nabla_{R}^{2} \Phi_{0}^{(2)}(R)+\nabla_{R}^{2} \Phi_{2}^{(2)}(R) P_{2}(\cos \Theta)+\frac{1}{R^{2}} \nabla_{\Theta}^{2} \Phi_{2}^{(2)}(R) P_{2}(\cos \Theta)=4 \pi G \rho(R) .
\end{gathered}
$$

Taking into account that $\xi(R, \Theta)=\xi_{0}(R)+$ $\xi_{2}(R) P_{2}(\cos \Theta)$, and collecting the corresponding terms we obtain the following Newtonian field equations of both static and rotating configurations

$$
\nabla_{R}^{2} \Phi^{(0)}(R)=4 \pi G \rho(R)
$$

$$
\begin{gathered}
\xi_{0}(R) \frac{d}{d R} \nabla_{R}^{2} \Phi^{(0)}(R)+\nabla_{R}^{2} \Phi_{0}^{(2)}(R)=0 \\
\xi_{2}(R) \frac{d}{d R} \nabla_{R}^{2} \Phi^{(0)}(R)+\nabla_{R}^{2} \Phi_{2}^{(2)}(R)- \\
-\frac{6}{R^{2}} \Phi_{2}^{(2)}(R)=0 .
\end{gathered}
$$


Two problems of major interest are to determine (1) the relation between mass and central density for a rotating star, and (2) the shape of the star. The differential equations for $\Phi_{0}^{(2)}(R)$, $\Phi_{2}^{(2)}(R), \xi_{0}(R)$ and $\xi_{2}(R)$, which will completely determine the equilibrium configuration, will now be given in forms suitable for solving these problems.

\section{Relation between mass and central density}

The relation between mass and central density may be determined from the $l=0$ equations alone.
The mass can be found from the term in $\Phi$ which is proportional to $1 / r$ at large distances. All components except $l=0$ vanish more strongly than this. Similarly near the origin all components of the density except $l=0$ vanish, so only the $l=0$ component contributes to the central density.

The total mass of the rotating configuration is given by the integral of the density over the volume. Writing this out in the $R, \Theta$ coordinates, expanding to order $\Omega^{2}$, and performing an integration by parts, one finds the change in mass $M^{(2)}$ of the rotating configuration from the non-rotating one. Thus the total mass is given by

$$
\begin{gathered}
M_{\text {tot }}=\int_{V} \rho(r, \theta) d V=\int_{V} \rho(r, \theta) r^{2} d r \sin \theta d \theta d \phi \\
=\left|r^{2} d r=(R+\xi)^{2}(d R+d \xi) \approx R^{2}\left(1+\frac{2 \xi}{R}\right)\left(1+\frac{d \xi}{d R}\right) d R=\left(1+\frac{2 \xi}{R}+\frac{d \xi}{d R}\right) R^{2} d R\right| \\
=\int_{V} \rho(R) R^{2} d R \sin \Theta d \Theta d \phi+\int_{V} \rho(R) R^{2}\left(\frac{2 \xi(R, \Theta)}{R}+\frac{d \xi(R, \Theta)}{d R}\right) d R \sin \Theta d \Theta d \phi .
\end{gathered}
$$

Performing the integration in the range of angles $0<\Theta<\pi$ and $0<\phi<2 \pi$ we obtain

$$
\begin{gathered}
M_{\text {tot }}(R)=M^{(0)}(R)+M^{(2)}(R), \\
M^{(0)}(R)=4 \pi \int_{0}^{R} \rho(R) R^{2} d R, \\
M^{(2)}(R)=4 \pi \int_{0}^{R} \rho(R) R^{2}\left(\frac{2 \xi_{0}(R)}{R}+\frac{d \xi_{0}(R)}{d R}\right) d R \\
=4 \pi \int_{0}^{R}\left(-\xi_{0}(R) \frac{d \rho(R)}{d R}\right) R^{2} d R,
\end{gathered}
$$

taking into account the following integrals

$$
\begin{gathered}
\int_{0}^{\pi} \sin \Theta d \Theta=2, \\
\int_{0}^{\pi} P_{2}(\cos \Theta) \sin \Theta d \Theta=0 .
\end{gathered}
$$

It is easy to show from the field equations and definitions of the masses

$$
\nabla_{R}^{2} \Phi^{(0)}(R)=\nabla^{2} \Phi^{(0)}(R)=4 \pi G \rho(R)
$$

$$
\begin{gathered}
\frac{d}{d R} \nabla_{R}^{2} \Phi^{(0)}(R)=\frac{d}{d R} \nabla^{2} \Phi^{(0)}(R)= \\
=4 \pi G \frac{d \rho(R)}{d R} \\
\frac{d M^{(0)}(R)}{d R}=4 \pi R^{2} \rho(R), \\
\frac{d M^{(2)}(R)}{d R}=4 \pi\left(-\xi_{0}(R) \frac{d \rho(R)}{d R}\right) R^{2},
\end{gathered}
$$

using the condition that $\Phi^{(0)}(R), \Phi_{0}^{(2)}(R) \rightarrow$ constant, as $R \rightarrow 0$, taking into account (22) the masses of both configurations can be expressed as

$$
\begin{aligned}
& \frac{G M^{(0)}(R)}{R^{2}}=\frac{d \Phi^{(0)}(R)}{d R}, \\
& \frac{G M^{(2)}(R)}{R^{2}}=\frac{d \Phi_{0}^{(2)}(R)}{d R},
\end{aligned}
$$

It is convenient to display the $l=0$ equation in a form in which it resembles the equation of hydrostatic equilibrium. To do this we make the definition 


$$
p_{0}^{*}(R)=\xi_{0}(R) \frac{d \Phi^{(0)}(R)}{d R}
$$

and taking derivative from (17) we obtain

$$
-\frac{d p_{0}^{*}(R)}{d R}+\frac{2}{3} \Omega^{2} R=\frac{G M^{(2)}(R)}{R^{2}},
$$

this equation along with

$$
\frac{d M^{(2)}(R)}{d R}=4 \pi R^{2} \rho(R) \frac{d \rho(R)}{d p} p_{0}^{*}(R),
$$

show the balance between the pressure, centrifugal, and gravitational forces per unit mass in the rotating star. The latter expression is written in terms of (16). To calculate the relation between mass and central density for the rotating star one now proceeds as follows: (1) Pick a value of the central density. Calculate the non-rotating configurationwith this central density. (2) Integrate equations (37) and (38) outward from the origin starting with the boundary condition which guarantees that the central density of the rotating configuration will have the same value.

$$
p_{0}^{*} \rightarrow \frac{1}{3} \Omega^{2} R^{2}, R \rightarrow 0 .
$$

(3) The value of $M^{(2)}(R)$ at the radius of the unperturbed star gives the change in mass of the rotating star over its non-rotating value for the same central density.

\section{tion \\ The shape of the star and numerical integra-}

The calculation of the shape of the rotating star involves the $l=2$ equations as well as those with $l=0$. If the surface of the non-rotating star has radius $a$ then equations (7) and (11) show that the equation for the surface of the rotating star has the form

$$
r(a, \Theta)=a+\xi_{0}(a)+\xi_{2}(a) P_{2}(\Theta) .
$$

The value of $\xi_{0}(a)$ is already determined in the $l=0$ calculation

$$
\xi_{0}(a)=\frac{a^{2}}{G M} p_{0}^{*}(a)
$$

Where $M=M^{(0)}(a)$ is the mass of the nonrotating configuration. However the determination of $\xi_{2}(R)$ from $l=2$ equations is not straightforward. So far, we have $l=2$ equations (18) and (23) representing hydrostatic equilibrium and field equations, respectively. Defining $\xi_{2}(R)$ from (18) and inserting it into (23) we have

$$
\begin{gathered}
\xi_{2}(R)=-\frac{R^{2}}{G M(R)}\left\{\frac{1}{3} \Omega^{2} R^{2}+\Phi_{2}^{(2)}(R)\right\} \\
\nabla_{R}^{2} \Phi_{2}^{(2)}(R)-\frac{6}{R^{2}} \Phi_{2}^{(2)}(R)=\frac{4 \pi R^{2}}{M(R)}\left\{\frac{1}{3} \Omega^{2} R^{2}+\right. \\
\left.+\Phi_{2}^{(2)}(R)\right\} \frac{d \rho(R)}{d R}
\end{gathered}
$$

where $M(R)=M^{(0)}(R)$ is the non-rotating mass for the sake of brevity. In order to solve the latter equation numerically one needs to rewrite it as first-order linear differential equations. Introducing new functions $\varphi=\Phi_{2}^{(2)}$ and $\chi$ equation (43) can be reduced to

$$
\begin{aligned}
\frac{d \chi(R)}{d R}=- & \frac{2 G M(R)}{R^{2}} \varphi(R)+\frac{8 \pi}{3} \Omega^{2} R^{3} G \rho(R), \\
\frac{d \varphi(R)}{d R}= & \left(\frac{4 \pi R^{2} \rho(R)}{M(R)}-\frac{2}{R}\right) \varphi(R)-\frac{2 \chi(R)}{G M(R)}+ \\
& +\frac{4 \pi}{3 M(R)} \rho(R) \Omega^{2} R^{4}
\end{aligned}
$$

The equations are solved for the derivatives so that they are in a form where their solution can be computed numerically by integrating outward from the origin. At the origin the solution must be regular. An examination of the equations shows that, as $R \rightarrow 0$,

$$
\varphi(R) \rightarrow A R^{2}, \chi(R) \rightarrow B R^{4},
$$

where $A$ and $B$ are any constants related by

$$
B+\frac{2 \pi}{3} G \rho_{c} A=\frac{2 \pi}{3} G \rho_{c} \Omega^{2},
$$

and where $\rho_{c}$ is the value of the density at the center of the star. The remaining constant in the solution is determined by the boundary condition that $\varphi(R) \rightarrow 0$ at large values of $R$. The constant is thus determined by joining the interior solution to that exterior solution which satisfies this boundary condition.

In the exterior region $(R>a)$ the solutions of the equations (44) and (45) are

$$
\varphi_{e x}(R)=\frac{K_{1}}{R^{3}}, \quad \chi_{e x}(R)=\frac{K_{1} G M^{(0)}}{2 R^{4}} .
$$


The interior solution to the equations (44) and (45) may be written as the sum of a particular solution and a homogeneous solution. The particular solution may be obtained by integrating the equations outward from the center with any values of $A$ and $B$ which satisfy (47). The homogeneous solution is then obtained by integrating the equations

$$
\begin{gathered}
\frac{d \chi_{h}(R)}{d R}=-\frac{2 G M(R)}{R^{2}} \varphi_{h}(R), \\
\frac{d \varphi_{h}(R)}{d R}=\left(\frac{4 \pi R^{2} \rho(R)}{M(R)}-\frac{2}{R}\right) \varphi_{h}(R)-\frac{2 \chi_{h}(R)}{G M(R)},
\end{gathered}
$$

with $A$ and $B$ related now by

$$
B+\frac{2 \pi}{3} G \rho_{c} A=0
$$

The general internal solution $(R<a)$ may then be written

$$
\begin{array}{r}
\varphi_{i n}(R)=\varphi_{p}(R)+K_{2} \varphi_{h}(R), \\
\chi_{i n}(R)=\chi_{p}(R)+K_{2} \chi_{h}(R) .
\end{array}
$$

By matching (48) and (52) at $(R=a)$ the constants $K_{1}$ and $K_{2}$ are defined. Thus $\varphi_{\text {in }}(R)$ is determined, hence $\xi_{2}(R)$ can be easily calculated from

$$
\xi_{2}(R)=-\frac{R^{2}}{G M(R)}\left\{\frac{1}{3} \Omega^{2} R^{2}+\Phi_{2(i n)}^{(2)}(R)\right\}
$$

\section{The ellipticity of the star}

The quantity defined by

$$
\epsilon(R)=-\frac{3}{2 R} \xi_{2}(R),
$$

is the ellipticity of the surface of constant density labeled by $R$ [15]. Using this expression and (42), then eliminating $\Phi_{2}^{(2)}$ in (43) one has the following equation for $\epsilon(R)$ :

$$
\begin{aligned}
& \frac{M(R)}{R} \frac{d^{2} \epsilon(R)}{d R^{2}}+\frac{2}{R} \frac{d M(R)}{d R} \frac{d \epsilon(R)}{d R}+ \\
& +\frac{2 d M(R)}{d R} \frac{\epsilon(R)}{R^{2}}-\frac{6 M(R) \epsilon(R)}{R^{3}}=0,
\end{aligned}
$$

or in a compact form it is written as follows

$$
\frac{d}{d R} \frac{1}{R^{4}} \frac{d}{d R}\left[\epsilon(R) M(R) R^{2}\right]=4 \pi \epsilon(R) \frac{d \rho(R)}{d R} .
$$

This equation is equivalent to Clairaut's equation [15]. Here both $M(R)$ and $\rho(R)$ are known functions of $R$. The ellipticity must be regular at small $R$, and equation (56) shows that it approaches a constant at $R=0$. With this boundary condition equation (56) may be integrated to find the shape of $\epsilon(R)$ but not its magnitude. To find the magnitude of $\epsilon(R)$ one needs to use (53). The procedure for the boundary condition at the surface in the previous section, together with the condition of regularity at the origin and the differential equation (56) uniquely determine the ellipticity of the surfaces of constant density as a function of the coordinate $R$.

\section{Quadrupole moment}

The Newtonian potential $\Phi(R, \Theta)$ outside the star $(R>a)$ will be written as before (see (10))

$$
\begin{gathered}
\Phi(R, \Theta)=\Phi^{(0)}(R)+\Phi_{0}^{(2)}(R)+ \\
+\Phi_{2}^{(2)}(R) P_{2}(\cos \Theta),
\end{gathered}
$$

where

$$
\begin{gathered}
\Phi^{(0)}(R)=-\frac{G M^{(0)}}{R}, \\
\Phi_{0}^{(2)}(R)=-\frac{G M^{(2)}}{R}, \\
\Phi_{2}^{(2)}(R)=\frac{K_{1}}{R^{3}} .
\end{gathered}
$$

In view of (25), the equation (57) is written as follows

$$
\Phi(R, \Theta)=-\frac{G M_{t o t}}{R}+\frac{K_{1}}{R^{3}} P_{2}(\cos \Theta)
$$

Thus constant $K_{1}$ can be written as $K_{1}=G Q$, where $Q$ is the mass quadrupole moment of the star. According to Hartle's definition $Q>0$ defines an oblate object, $Q<0$ defines a prolate object.

\section{Moment of inertia}

Similarly to the total mass of the star the total moment of inertia can be calculated

$$
I_{\text {tot }}=\int_{V} \rho(r, \theta)(r \sin \theta)^{2} d V=\int_{V} \rho(r, \theta) r^{4} d r \sin ^{3} \theta d \theta d \phi
$$




$$
\begin{gathered}
=\left|\begin{array}{c}
r^{4} d r=(R+\xi)^{4}(d R+d \xi) \approx R^{4}\left(1+\frac{4 \xi}{R}\right)\left(1+\frac{d \xi}{d R}\right) d R \\
=\left(1+\frac{4 \xi}{R}+\frac{d \xi}{d R}\right) R^{4} d R
\end{array}\right| \\
=\int_{V} \rho(R) R^{4} d R \sin ^{3} \Theta d \Theta d \phi+\int_{V} \rho(R) R^{4}\left(\frac{4 \xi(R, \Theta)}{R}+\frac{d \xi(R, \Theta)}{d R}\right) d R \sin ^{3} \Theta d \Theta d \phi
\end{gathered}
$$

Performing integration in the range of angles $0<\Theta<\pi$ and $0<\phi<2 \pi$ we obtain

$$
\begin{gathered}
I_{\text {tot }}(R)=I^{(0)}(R)+I^{(2)}(R), \\
I^{(0)}(R)=\frac{8 \pi}{3} \int_{0}^{R} \rho(R) R^{4} d R, \\
I^{(2)}(R)=\frac{8 \pi}{3} \int_{0}^{R} \rho(R) R^{4}\left(\frac{d \xi_{0}}{d R}-\frac{1}{5} \frac{d \xi_{2}}{d R}\right. \\
\left.+\frac{4}{R}\left[\xi_{0}-\frac{1}{5} \xi_{2}\right]\right) d R \\
=\frac{8 \pi}{3} \int_{0}^{R}\left(\left[\frac{1}{5} \xi_{2}(R)\right.\right. \\
\left.\left.-\xi_{0}(R)\right] \frac{d \rho}{d R}\right) R^{4} d R,
\end{gathered}
$$

taking into account the following integrals

$$
\begin{gathered}
\int_{0}^{\pi} \sin ^{3} \Theta d \Theta=\frac{4}{3^{\prime}} \\
\int_{0}^{\pi} P_{2}(\cos \Theta) \sin ^{3} \Theta d \Theta=-\frac{4}{15} .
\end{gathered}
$$

The definition of the moment of inertia for slowly rotating relativistic stars can be found in [14].

\section{Summary: static case}

To determine the relation between mass and central density one now proceeds as follows. (1) Assign the equation of state $p=p(\rho)$ (polytrope, tabulated, etc.). (2) Choose the value of the central density $\rho(R=0)=\rho_{c}$. Calculate the mass and pressure from the Newtonian field equation and equation of hydrostatic equilibrium with the regularity condition at the center $M^{(0)}(R=0)=0$.

$$
\left\{\begin{array}{c}
\frac{d M^{(0)}(R)}{d R}=4 \pi R^{2} \rho(R), \\
\frac{d p^{(0)}(R)}{d R}=-\rho(R) \frac{G M^{(0)}(R)}{R^{2}},
\end{array}\right.
$$

The gravitational potential of a non-rotating star is defined by

$$
\frac{d \Phi^{(0)}(R)}{d R}=\frac{G M^{(0)}(R)}{R^{2}}=-\frac{1}{\rho(R)} \frac{d p^{(0)}(R)}{d R}
$$

On the surface the pressure must vanish $p^{(0)}(R=a)=0$.

\section{Summary: $l=0$ equations}

Choose the value of the angular velocity of the star. For instance, as a test value take

$$
\Omega=\sqrt{\frac{G M^{(0)}(a)}{a^{3}}}
$$

Integrate the couplet equations

$$
\left\{\begin{array}{c}
\frac{d p_{0}^{*}(R)}{d R}=\frac{2}{3} \Omega^{2} R-\frac{G M^{(2)}(R)}{R^{2}} \\
\frac{d M^{(2)}(R)}{d R}=4 \pi R^{2} \rho(R) \frac{d \rho(R)}{d R} p_{0}^{*}(R),
\end{array},\right.
$$

out from the origin with boundary conditions that as $R \rightarrow 0$

$$
p_{0}^{*}(R) \rightarrow \frac{1}{3} \Omega^{2} R^{2}, M^{(2)}(R) \rightarrow 0 .
$$

These boundary conditions guarantee that the central density of the rotating and non-rotating configurations are the same.

Summary: $l=2$ equations 


\section{A. Particular Solution}

Integrate equations

$$
\left\{\begin{array}{c}
\frac{d \chi(R)}{d R}=-\frac{2 G M(R)}{R^{2}} \varphi(R)+\frac{8 \pi}{3} \Omega^{2} R^{3} G \rho(R) \\
\frac{d \varphi(R)}{d R}=\left(\frac{4 \pi R^{2} \rho(R)}{M(R)}-\frac{2}{R}\right) \varphi(R)-\frac{2 \chi(R)}{G M(R)}+\frac{4 \pi}{3 M(R)} \rho \Omega^{2} R^{4}
\end{array}\right.
$$

outward from the center with arbitrary initial conditions satisfying equations

$$
\begin{gathered}
\varphi(R) \rightarrow A R^{2}, \quad \chi(R) \rightarrow B R^{4}, \\
B+\frac{2 \pi}{3} G \rho_{c} A=\frac{2 \pi}{3} G \rho_{c} \Omega^{2},
\end{gathered}
$$

where $A$ and $B$ are arbitrary constants. Set, for example, $A=1$ and define $B$ from the above algebraic equation. This determines particular solutions $\varphi_{p}(R)$ and $\chi_{p}(R)$.

\section{B. Homogeneous Solution}

Integrate the homogeneous equations

$$
\left\{\begin{array}{c}
\frac{d \chi_{h}(R)}{d R}=-\frac{2 G M(R)}{R^{2}} \varphi_{h}(R) \\
\frac{d \varphi_{h}(R)}{d R}=\left(\frac{4 \pi R^{2} \rho(R)}{M(R)}-\frac{2}{R}\right) \varphi_{h}(R)-\frac{2 \chi_{h}(R)}{G M(R)}
\end{array}\right.
$$

outward from the center with arbitrary initial conditions satisfying the equations

$$
\begin{gathered}
\varphi_{h}(R) \rightarrow A R^{2}, \quad \chi_{h}(R) \rightarrow B R^{4}, \\
B+\frac{2 \pi}{3} G \rho_{c} A=0
\end{gathered}
$$

Set $A=1$ and $B$ is given by the above equation. This determines particular solutions $\varphi_{h}(R)$ and $\chi_{h}(R)$. Thus interior solution is defined by the sum of the particular and the homogeneous solution

$$
\begin{gathered}
\varphi_{i n}(R)=\varphi_{p}(R)+K_{2} \varphi_{h}(R), \\
\chi_{i n}(R)=\chi_{p}(R)+K_{2} \chi_{h}(R) .
\end{gathered}
$$

\section{Matching with the Exterior Solutions}

The exterior solutions

$$
\varphi_{e x}(R)=\frac{K_{1}}{R^{3}}, \quad \chi_{e x}(R)=\frac{K_{1} G M^{(0)}}{2 R^{4}} .
$$

By matching (77) and (76) at $(R=a)$

$$
\begin{gathered}
\varphi_{\text {ex }}(R=a)=\varphi_{\text {in }}(R=a), \\
\chi_{\text {ex }}(R=a)=\chi_{\text {in }}(R=a) .
\end{gathered}
$$

constants $K_{1}$ and $K_{2}$ are defined. The surface of the rotating configuration, polar $r_{p}$ and equatorial $r_{e}$ radii are given by

$$
\begin{gathered}
r(a, \Theta)=a+\xi_{0}(a)+\xi_{2}(a) P_{2}(\Theta), \\
r_{p}=r(a, 0)=a+\xi_{0}(a)+\xi_{2}(a), \\
r_{e}=r(a, \pi / 2)=a+\xi_{0}(a)-\xi_{2}(a) / 2
\end{gathered}
$$

\section{Conclusion}

Equations have been developed for calculating the structure of slowly rotating classical stars in hydrostatic equilibrium. In particular, prescriptions have been given to find the relation between mass and central density, the shapes of rotating stars.

The equation which determines the relation between mass and central density takes the form of an equation of hydrostatic equilibrium. It enforces the balance of pressure, gravitational, and centrifugal forces correctly to order $\Omega^{2}$ in the angular velocity. In this order the surface of constant density are spheroids whose ellipticity varies from zero at the center of the star to the ellipticity which describes the shape of the star at the surface. The ellipticity, $\epsilon(R)$, as a function of radius is determined by Clairaut's differential equation for this quantity.

Both the equations which determine the relation between mass and central density and those which determine the ellipticity are systems of ordinary, first-order linear differential equations whose solution may be obtained by numerical integration.

The equations which determine the moment of inertia and the quadrupole moment of the rotating star have also been defined. The product of the moment of inertia and the angular velocity defines the angular momentum of the star. 
All these quantities are very crucial in describing the equilibrium configurations of uniformly rotating stars and planets.

\section{Acknowledgements}

The author expresses his deep gratitude to his students: Balgimbekov Galymdin, Abutalip Marzhan, Kalymova Zhanerke and Suleymanova Shara- ra for the technical assistance. This work was supported in part by the grant No. IPC-30 of the MES RK.

\section{Appendix}

In the paper we used frequently the Taylor series of function $f\left(x_{0}+\Delta x\right)$

$$
f\left(x_{0}+\Delta x\right)=f\left(x_{0}\right)+\Delta x f^{\prime}\left(x_{0}\right)+\frac{(\Delta x)^{2}}{2 !} f^{\prime \prime}\left(x_{0}\right)+\frac{(\Delta x)^{3}}{3 !} f^{\prime \prime \prime}\left(x_{0}\right)+\cdots
$$

where $\Delta x=x-x_{0}$. Moreover we had the following algebraic manipulations

$$
\begin{gathered}
r=R+\xi \\
\frac{1}{r}=\frac{1}{R+\xi} \approx \frac{1}{R}\left(1-\frac{\xi}{R}\right), \\
d r=d R+d \xi=\left(1+\frac{d \xi}{d R}\right) d R, \\
\frac{d r}{d R}=\left(1+\frac{d \xi}{d R}\right), \\
\frac{d R}{d r} \approx\left(1-\frac{\xi}{R}\right) .
\end{gathered}
$$

The Newtonian field equations for $\Phi^{(0)}(r)$ in $R, \Theta$ coordinates will have the form

$$
\nabla_{r}^{2} \Phi^{(0)}(r) \approx \nabla_{R}^{2} \Phi^{(0)}(R)+\xi(R, \Theta) \frac{d}{d R} \nabla_{R}^{2} \Phi^{(0)}(R)
$$

Now let us see technical details

$$
\begin{gathered}
\nabla_{r}^{2} \Phi^{(0)}(r)=\left[\frac{d^{2}}{d r^{2}}+\frac{2}{r} \frac{d}{d r}\right] \Phi^{(0)}(r)=\left[\frac{d R}{d r} \frac{d}{d R} \frac{d R}{d r} \frac{d}{d R}+\frac{2}{R+\xi} \frac{d R}{d r} \frac{d}{d R}\right] \Phi^{(0)}(R+\xi) \\
\approx\left[\left(1-\frac{d \xi}{d R}\right) \frac{d}{d R}\left(1-\frac{d \xi}{d R}\right) \frac{d}{d R}+\frac{2}{R}\left(1-\frac{\xi}{R}\right)\left(1-\frac{d \xi}{d R}\right) \frac{d}{d R}\right]\left[\Phi^{(0)}(R)+\xi \frac{d \Phi^{(0)}(R)}{d R}\right] \\
\approx\left[\left(1-\frac{d \xi}{d R}\right)\left\{-\frac{d^{2} \xi}{d R^{2}} \frac{d}{d R}+\left(1-\frac{d \xi}{d R}\right) \frac{d^{2}}{d R^{2}}\right\}+\frac{2}{R}\left(1-\frac{\xi}{R}-\frac{d \xi}{d R}\right) \frac{d}{d R}\right]\left[\Phi^{(0)}(R)+\xi \frac{d \Phi^{(0)}(R)}{d R}\right] \\
\approx\left[\frac{d^{2}}{d R^{2}}+\frac{2}{R} \frac{d}{d R}-\frac{2 d \xi}{d R} \frac{d^{2}}{d R^{2}}-\frac{2}{R}\left(\frac{\xi}{R}+\frac{d \xi}{d R}+\frac{R}{2} \frac{d^{2} \xi}{d R^{2}}\right) \frac{d}{d R}\right]\left[\Phi^{(0)}(R)+\xi \frac{d \Phi^{(0)}(R)}{d R}\right] \\
\approx\left[\frac{d^{2}}{d R^{2}}+\frac{2}{R} \frac{d}{d R}-\frac{2 d \xi}{d R} \frac{d^{2}}{d R^{2}}-\frac{2}{R}\left(\frac{\xi}{R}+\frac{d \xi}{d R}+\frac{R}{2} \frac{d^{2} \xi}{d R^{2}}\right) \frac{d}{d R}\right] \Phi^{(0)}(R)+\left[\frac{d^{2}}{d R^{2}}+\frac{2}{R} \frac{d}{d R}\right] \xi \frac{d \Phi^{(0)}(R)}{d R}
\end{gathered}
$$




$$
\begin{gathered}
=\left[\frac{d^{2}}{d R^{2}}+\frac{2}{R} \frac{d}{d R}-\frac{2 d \xi}{d R} \frac{d^{2}}{d R^{2}}-\frac{2}{R}\left(\frac{\xi}{R}+\frac{d \xi}{d R}+\frac{R}{2} \frac{d^{2} \xi}{d R^{2}}\right) \frac{d}{d R}\right] \Phi^{(0)}(R)+\frac{d \Phi^{(0)}(R)}{d R}\left[\frac{d^{2}}{d R^{2}}+\frac{2}{R} \frac{d}{d R}\right] \xi \\
+\xi\left[\frac{d^{2}}{d R^{2}}+\frac{2}{R} \frac{d}{d R}\right] \frac{d \Phi^{(0)}(R)}{d R}+\frac{2 d \xi}{d R} \frac{d^{2} \Phi^{(0)}(R)}{d R^{2}} \\
=\left[\frac{d^{2}}{d R^{2}}+\frac{2}{R} \frac{d}{d R}-\frac{2 \xi}{R^{2}} \frac{d}{d R}\right] \Phi^{(0)}(R)+\xi\left[\frac{d^{2}}{d R^{2}}+\frac{2}{R} \frac{d}{d R}\right] \frac{d \Phi^{(0)}(R)}{d R} \\
=\left[\frac{d^{2}}{d R^{2}}+\frac{2}{R} \frac{d}{d R}\right] \Phi^{(0)}(R)+\xi \frac{d}{d R}\left[\frac{d^{2}}{d R^{2}}+\frac{2}{R} \frac{d}{d R}\right] \Phi^{(0)}(R)=\nabla_{R}^{2} \Phi^{(0)}(R)+\xi \frac{d}{d R} \nabla_{R}^{2} \Phi^{(0)}(R)
\end{gathered}
$$

\section{References}

1 Meynet, G., Maeder, A. Stellar evolution with rotation. V. Changes in all the outputs of massive star models // Astronomy and Astrophysics. 2000. -Vol.361. - P.101-120.

2 Meynet, G., Maeder, A. Stellar evolution with rotation. X. Wolf-Rayet star populations at solar metallicity // Astronomy and Astrophysics. 2003. - Vol.404.- P. 975-990.

3 Ekstrom S., Meynet G., Chiappini C., Hirschi R., Maeder A. Effects of rotation on the evolution of primordial stars // Astronomy and Astrophysics.- 2008. - Vol. 489.- Issue 2.- P.685-698.

4 Chandrasekhar S. The Maximum Mass of Ideal White Dwarfs // Astrophysical Journal. -1931. - Vol. 74. - P. 81-82.

5 Chandrasekhar S., Roberts P. On Highly Rotating Polytropes. II // Astrophysical Journal.-1963. - Vol. 138. - P. 809.

6 Stergioulas N. Rotating Stars in Relativity // Living Reviews in Relativity. - 2003. - Vol. 6. -P. 3.

7 Hartle J. B. Slowly Rotating Relativistic Stars. I. Equations of Structure // Astrophysical Journal. -1967. - Vol. 150. - P. 1005.

8 Bondi H. Massive Spheres in General Relativity // Proceedings of the Royal Society of Lon- don. Series A, Mathematical and Physical Sciences. -1964. - Vol. 284. - Issue 1390. - P. 303-317.

9 Buchdahl H. General Relativistic Fluid Spheres // Physical Review. - 1959. - Vol. 116. Issue 4. - P. 1027-1034.

10 Chandrasekhar S. Introductionto the Study of Stellar Structure. Chicago: University of Chicago Press. - 1939.

11 Harrison B., Thorne K., Wakano M., Wheeler J. Gravitation Theory and Gravitational Collapse. Cambridge: Cambridge University Press. $-1965$.

12 Hartle, J. B., Sharp D. H. Variational Principle for the Equilibrium of a Relativistic, Rotating Star // Astrophysical Journal. - 1967. - Vol.147. P. 317.

13 Jeffreys H. The Earth. Cambridge: Cambridge University Press. - 1959.

14 Hartle J. B. Slowly Rotating Relativistic Stars. IX: Moments of Inertia of Rotationally Distorted Stars // Astrophysics and Space Science. 1973. - Vol. 24. - P. 385-405.

15 Chandrasekhar S., Roberts P. The Ellipticity of a Slowly Rotating Configuration // Astrophysical Journal.-1963. - Vol. 138. - P. 801. 